\title{
¿Por qué demoran en publicarse los manuscritos en la Revista Médica de Chile?
}

\author{
What determines the time-lag \\ for publications in \\ Revista Médica de Chile?
}

The number of manuscripts submitted for publication in Revista Médica de Chile has increased steadily. Consequently, the number of external experts that generously contribute in the peer review process has also been enlarged. When the manuscripts return to the authors with specific requests to be improved, according to the criticisms raised by their reviewers or the editors, more time is added until a corrected version is received and accepted. After acceptance, the manuscripts are programmed by the editors to be included in future monthly issues of the Revista. The number of manuscripts that can be included in an issue is limited by the number of pages accorded between the publishers and the owner institution. Therefore, an additional timelag occurs: research articles and case reports may be published 6-8 months after they were finally accepted, while reviews and special articles have to wait even more. As experienced in prestigious journals worldwide, a computational program to handle the editorial process through the Internet is needed, but it is not yet available in Spanish language. Although it should fasten communications and facilitate the authors a rapid insight in the stages of the editorial process where their manuscripts are, the increasing number of submissions, the current acceptance rate and the limitation in space will still retard the publications once they are accepted (Rev Méd Chile 2006; 134: 811-12).

(Key-words: Journalism, medical; Manuscripts, medical; Peer review; Periodicals)

$F_{y}^{n}$ recuentemente recibimos consultas telefónicas que desean saber en qué etapa del proceso editorial se encuentra su manuscrito. Mientras el manuscrito está en etapa de revisión por pares, un factor cronológico importante es la demora en obtener respuestas de los revisores externos. A ello se agrega el tiempo que necesiten los autores para re-enviar una versión corregida según lo solicitado por los revisores y los editores. Una vez que los autores han recibido una carta en que se les comunica que el manuscrito corregido "... ha sido considerado satisfactorio en sus aspectos científicos. Su aprobación definitiva y su programación requerirán una revisión técnica por los Editores", se inicia una espera que provoca nuevas inquietudes en muchos autores y se reinician sus consultas.

Hay etapas del proceso editorial que necesariamente son confidenciales, tales como la revisión por pares. Pero la cronología del proceso total, desde la recepción de un manuscrito hasta su publicación, no necesita ser confidencial y sería ideal que los autores pudieran acceder periódicamente a la información sobre lo que está ocurriendo con sus manuscritos. Para que esto se implemente en una revista médico-científica, se necesita que los manuscritos se manejen mediante un programa computacional al que deben adaptarse todos los manuscritos, desde que son enviados a la revista hasta que son entregados a la imprenta. 
Recientemente, los Editores de Croatian Medical Journal comentaron "Nuestro antiguo sistema de papel y carpetas para mantener el registro de los manuscritos recibidos funcionó bien mientras manejábamos menos de 200 artículos por año. Desde el momento en que excedimos este volumen se hizo muy difícil seguir el rastro de lo que pasa, especialmente del proceso de revisión editorial que a menudo incluye a tres revisores externos y un revisor estadístico. El año pasado, cuando el número de manuscritos recibidos sobrepasó 300, esto se hizo casi imposible"1. El año pasado nuestra Revista sobrepasó con creces el número límite de "manejabilidad manual" que se detectó en la revista europea aludida.

Croatian Medical Journal cuenta con un sistema electrónico para el manejo de los manuscritos y para la intercomunicación entre secretarias, editores, autores, revisores externos e impresores. Su sistema fue adaptado por la Facultad de Ingenieńa Eléctrica y Computación de su Universidad, del que habían diseñado para su propia revista ${ }^{1}$. En nuestra situación, no tenemos todavía un programa computacional adecuado, en idioma castellano. Esperamos contar con la ayuda de CONICYT y su programa SciELO, donde están ensayando un modelo para las revistas chilenas. Una de las ventajas de tales programas es que permiten a los autores el seguimiento de sus manuscritos durante el proceso editorial, mediante consultas electrónicas que no intemumpen el trabajo de las secretarias ni los Editores.

Pero, ¿será ésa una respuesta satisfactoria a la pregunta que encabeza esta Editorial?

El proceso a que se someten los manuscritos enviados a la Revista Médica de Chile, desde que se reciben en Secretaría, fue descrito en enero de $2004^{2}$ y actualizado en enero de $2006^{3}$. Ambas Editoriales analizaron las etapas de este proceso y el tiempo que demora. Además, informaron que cada número mensual de la Revista tiene un cupo máximo de páginas (actualmente 136) y éste es el factor crucial que determina la cantidad de ma-

\section{REFERENCIAS}

1. Marucic A, Marusic M. Double Life of Medical Journals: Dr Paper and Mr Web (Editorial). Croat Med J 2006; 47: 4-6.

2. Reyes H, Palma J, Andresen M. El manejo editorial nuscritos que pueden incluirse. Por ello, en las "Instrucciones a los Autores" se les solicita que limiten la extensión de los manuscritos (número de palabras del texto y número de referencias), para dar cabida con la mayor rapidez posible a los que sean aceptados.

Después de ser revisados por expertos externos y haberse aprobado una versión corregida, los manuscritos son programados por los Editores considerando como criterio prioritario su secuencia según la fecha de aceptación. La fecha de recepción de la primera versión del manuscrito es un criterio adicional, pero no prioritario para su programación.

En el primer trimestre de 2006, los manuscritos clasificados como "Artículos de Investigación" y "Casos Clínicos" se publicaron 6 a 8 meses después de haber sido aceptados. Para los manuscritos clasificados como "Artículos de Revisión” y “Artículos Especiales" la espera fluctuó entre 7 y 11 meses.

La demanda por publicar en la Revista Médica de Chile ha alcanzado tasas inéditas. Los revisores hacen indicaciones que consideran razonables para ayudar a los autores a mejorar sus manuscritos. Los editores insisten a los autores que cumplan con la formalidad, brevedad y presentación que comesponden al estilo de la Revista y con normas aceptadas internacionalmente por revistas de naturaleza similar. Los autores hacen su mejor esfuerzo y una buena proporción de los manuscritos son aceptados. Pero todo este proceso toma tiempo ("T.T.T.: Things Take Time" 2) y el espacio es limitado. La adopción de un programa computacional para recibir y manejar los manuscritos será, sin duda, un progreso indispensable que todos debemos propiciar. Pero no esperemos que con ello los plazos de publicación se acorten sustantivamente, porque persiste la limitación del espacio de cabida para los manuscritos que sean aceptados.

\section{Humberto Reyes B.}

\section{Editor}

\section{Max Andresen H, Joaquín Palma $H$.} Editores Asociados

de los manuscritos recibidos en la Revista Médica de Chile (Editorial). Rev Méd Chile 2004; 132: 7-10.

3. Reyes H, Andresen M, Palma J. El creciente desafío del proceso editorial en la Revista Médica de Chile. Rev Méd Chile 2006; 134: 7-11. 